\title{
Fuzzy based prediction schema framework for IoT based indoor environmental monitoring
}

\begin{abstract}
In recent times, rapid introduction of the Internet of Things (IoT) services in smart environment domain has happened. This has introduced heterogeneous device and data types that lack proper mechanism for joint execution of tasks from an application perspective. In this paper, we propose an integrated indoor environmental monitoring system implemented with a novel IoT framework. It is enabled via fuzzy-based rule schema to resolve the heterogeneity of environmental data in smart home environment. The novel aspect of the framework is that it is developed with a modular repository together with decision-making modules. Our experiments prove this approach to be a viable IoT solution for smart home indoor environment.
\end{abstract}

Keyword: Fuzzy based prediction; IoT; Smart home; Indoor environmental monitoring 